\title{
Multiple Cerebrovascular Occlusion With Hypothyroidism: A Powerful Compensation
}

\author{
Yi Bao a, b, Quanying Liu ${ }^{\mathrm{a}}$, Shihao Xua ${ }^{\mathrm{a}}$, Yuchen Wanga, \\ Xiaodong Liu ${ }^{\mathrm{a}}$, Guangjian $\mathrm{Liu}^{\mathrm{a}}$
}

\begin{abstract}
Cerebral vascular occlusion can often cause severe neurological deficits. This study aims to describe a case characterized by multiple large vessel occlusions with hypothyroidism and without clinical symptoms. The patient's blood vessel condition, compensatory pathway and perfusion situation were understood through medical history inquiry, neurological physical examination, combined with blood lipid, blood glucose, electrocardiogram, transcranial Doppler (TCD), brain computed tomography (CT), cerebral angiography, cerebral perfusion imaging and other examinations. The main symptoms of the patient were dizziness and headache, with no symptoms of neurological function loss. Digital subtraction angiography (DSA) showed multiple large vessel occlusions in the head and neck: the initial part of the left internal carotid artery, the M1 segment of the left middle cerebral artery, the V2 segment of the right vertebral artery, and the V3 terminal segment of the left vertebral artery. According to CT perfusion (CTP) examination, the blood flow (BF) value of the left temporoparietal occipital lobe was lower than that of the contralateral side, the mean blood flow passage time value was increased, and the cerebral blood volume (BV) was slightly higher than that of the contralateral side. Cerebral vascular occlusion is affected by many factors, hypothyroidism can accelerate the process of atherosclerosis, and in severe cases multiple large vessel occlusions may occur. Powerful compensatory mechanisms can reduce the incidence of stroke.
\end{abstract}

Keywords: Cerebrovascular occlusion; Hypothyroidism; Compensation; DSA

\section{Introduction}

Ischemic stroke is characterized by sudden disturbance of consciousness, speech disorders, and paralysis of the limbs. It has

Manuscript submitted April 20, 2019, accepted May 17, 2019

aDepartment of Neurology, Taihe Hospital Affiliated to Hubei University of Medicine, Shiyan City, Hubei Province, China

${ }^{b}$ Corresponding Author: Yi Bao, Department of Neurology, Taihe Hospital Affiliated to Hubei University of Medicine, Shiyan City, Hubei Province 442000, China.Email: karlbaoyi@163.com

doi: https://doi.org/10.14740/jmc3303 the features of high morbidity and mortality, which poses a great threat to human health and life [1].

It is well known that atherosclerosis is one of the important causes of arterial stenosis and even occlusion. The lesions often involve the large and medium muscular arteries [2, 3]. Major risk factors include hypertension, hyperlipidemia, heavy smoking, diabetes, obesity and genetic factors [4-6]. Patients with hypothyroidism often have elevated blood lipids, creatine phosphokinase activity, and a low glucose tolerance curve, which often accelerate the progression of cerebrovascular disease [7, 8].

In this case, the patient had a history of hypothyroidism, and the main symptoms were dizziness, headache, and no loss of neurological function. Digital subtraction angiography (DSA) revealed multiple vascular occlusions in the head and neck: the starting of the left internal carotid artery (ICA), the left middle cerebral artery M1 segment, the right side of the vertebral artery V2, V3 end on the left side of the vertebral artery occlusion. In view of the discrepancy between mild symptoms and severe vascular occlusion, we consider that the patient has a strong compensatory mechanism and analyze the reasons.

\section{Case Report}

A 65-year-old man was admitted to the hospital for 3 months due to dizziness and headache. The main manifestations were: dizziness, unstable gait, the right temporal jump pain that spread to the top of the head and contralateral side and attack 2 - 3 times a day, for more than $10 \mathrm{~min}$ each time. In severe cases, it was accompanied by nausea and vomiting. It was aggravated when tired, relieved after rest, with no fever during the course of the disease, no blurred vision, no diplopia, no cough when drinking water, and no limb numbness. He had a history of hypertension for more than 20 years, up to $180 / 90 \mathrm{~mm} \mathrm{Hg}$; a history of type 2 diabetes for 2 years; a history of coronary heart disease for more than 10 years; coronary artery stenting was performed in 2015; a history of hypothyroidism for 4 years, and had long-term thyroid treatment. He had been treated in local hospitals for many times, and his dizziness and headache had not been improved significantly. He came to the department of neurology of our hospital for further diagnosis and treatment.

\section{Routine examinations and treatments}

Physical examination showed no positive signs of internal 

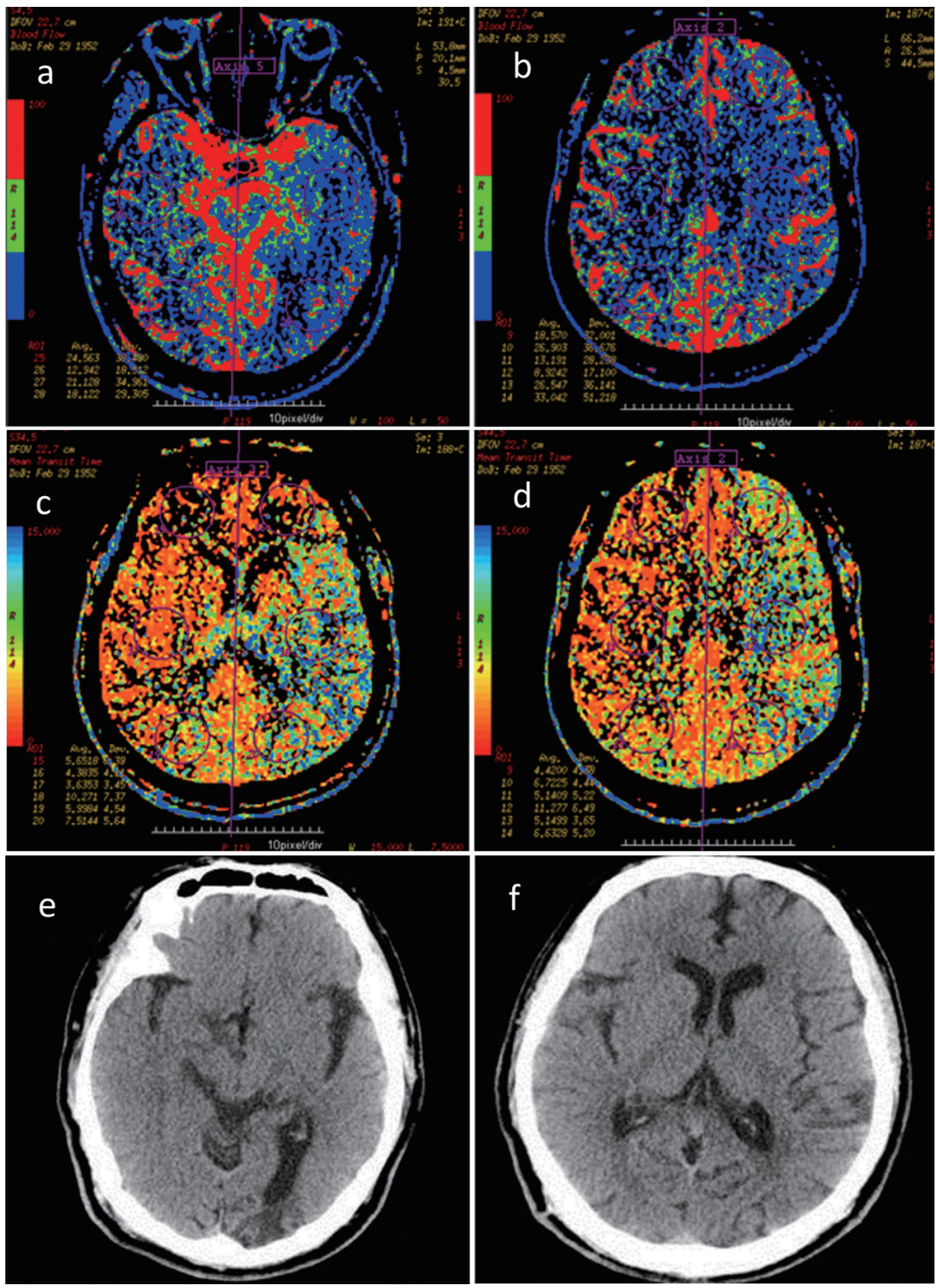

Figure 1. (a-d) According to CTP, BF in left temporoparietal occipital lobe was lower than that in the contralateral side, blood flow MTT was higher, and cerebral BV was slightly higher than that in the contralateral side. (e, f) According to CT, there is no new cerebral infarction. BF: blood flow; MTT: mean transit time; BV: blood volume.

medicine system and nervous system. The general examination and other investigations were normal. No obvious lesion was found in the brain computed tomography (CT) of the patient, as shown in Figure 1e, d. Using two-dimensional ultrasound transcranial Doppler (TCD), bilateral carotid arteries and their branches showed extensive atherosclerotic plaque formation, no blood flow (BF) signals in the left ICA opening segment and extracranial segment, and the right common carotid artery (RCCA) dilation. The left middle cerebral artery, the anterior artery had low flow velocity and low resistance type spectrum change and the abnormal flow rate increased with whirlpool signal. When the RCCA was pressed, the BF velocity of each branch on the left decreased, and severe stenosis or occlusion occurred from end of left ICA to left ICA segment and blood was supplied from the right to the left. The mean velocity of each branch artery in the posterior circulation was low. The intracranial arterial resistance spectrum increased. In terms of treatment, aspirin was given to prevent platelet aggregation, rosuvastatin to reduce blood lipid, control blood glucose and blood pressure, and supplement of Euthyrox to treat hypothy- 
roidism and protect stomach, and so on.

\section{DSA examination of cerebral vascular condition}

The dizziness and headache of the patient were not significantly improved. In order to further clarify the cerebral vascular condition, the whole cerebral angiography was performed. Results of cerebral vascular DSA showed: bilateral common carotid artery, subclavian artery and extracranial vertebral artery were tortuous, bilateral vertebral artery V1-V2 segment segmental stenosis was $30 \%-50 \%$ and bilateral carotid sinus plaque was observed. The left ICA was occluded at the beginning, the left external carotid artery showed rough vascular wall and no branches were seen to supply blood into left ICA. The right ICA intracranial arteriosclerosis, the right middle cerebral artery and anterior cerebral artery wall were rough, with no obvious stenosis. The anterior communicating artery was opened, and right ICA system compensated blood supply to left anterior cerebral artery and middle cerebral artery through the anterior communicating artery. The M1 segment of left middle cerebral artery was occluded, with local capillary hyperplasia. Low signal was at the far end of M2 segment of left middle cerebral artery. The left anterior cerebral artery was less smooth without obvious stenosis and its meningeal branch supplied blood to left middle cerebral artery. The right posterior communicating artery was not opened. The right external carotid artery wall was not smooth. The V2 segment of right vertebral artery was occluded, and muscular branch and the branch of right cervical artery were anastomosed with anterior spinal artery. The blood supply to V4 segment of right vertebral artery was compensated by the anterior spinal artery, the signal of basilar artery and bilateral posterior cerebral artery was low. The signal of each venous phase was good (Fig. 2).

\section{Brain CTP examination}

According to the severe occlusion of cerebral blood vessels, it is necessary to know whether cerebral blood perfusion is sufficient, and brain CT perfusion imaging (CTP) is performed. Brain CTP test results were as follows: the BF value of left temporoparietal occipital lobe was lower than that of the contralateral side, BF mean transit time (MTT) value was higher, and cerebral blood volume (BV) was slightly higher than that in the contralateral side. No obvious abnormality was found in the remaining perfusion area. Therefore, the perfusion of left temporoparietal occipital lobe was decreased (Fig. 1).

\section{Treatment course and prognosis}

The left temporoparietal occipital lobe BF perfusion decreased and the risk of stroke was high. Based on consultation with neurosurgery, it is recommended that antiplatelet therapy be continued and, if necessary, vascular bypass or interventional therapy be performed to improve cerebral BF. After full disclosure of the benefits and risks, the patient and his families requested discharge. He was instructed to eat a diet low in salt and fat, avoiding bad living habits, taking medicine regularly, keeping a happy mood and exercising moderately. After discharge, combined Plavix (75 mg) with Bayaspirin $(100 \mathrm{mg})$ was used to inhibit the platelet aggregation every day. Regular review, if necessary, checks the cerebral vascular DSA and a neurosurgery can be performed timely once there are surgical indications.

\section{Discussion}

Cerebrovascular occlusion is a serious threat to the patient's life and clinical manifestations are closely related to the responsible blood supply area. According to the ICA system, the middle cerebral artery is the most common, with hemiplegia, partial sensory disturbance, hemianopia and psychiatric symptoms. There is aphasia in dominant hemisphere lesions $[9,10]$. Vertebrobasilar artery system can be manifested as high fever, coma, vertigo, nystagmus, transient amaurosis, tinnitus, deafness, limb paralysis, cerebellar ataxia and Horner's sign. Acute complete occlusion can rapidly endanger life, presenting as atresia syndrome sometimes [11]. The occluded vessels in this patient were: the initial part of left ICA, M1 segment of left middle cerebral artery, V2 segment of right vertebral artery, and V3 terminal segment of left vertebral artery, but the clinical manifestations as followed were not observed, which is one of the significant characteristics of this case.

There are many factors involved in the occurrence and development of cerebral vascular occlusion, among which dyslipidemia is vital. The patient has many risk factors for atherosclerosis, such as age, known hypertension with high base line blood pressure, and diabetes. Hypothyroidism is one of the risk factors. Combined with literature reports [7, 8], in terms of low-density lipoprotein cholesterol (LDL-C), hyperlipidemia (triglyceride, TG), total cholesterol (TC), homocysteine, hemorheology, hemodynamics and other indicators, patients with hypothyroidism or subclinical hypothyroidism are significantly different from those with simple ischemic cerebrovascular disease, which will accelerate atherosclerosis and may have an impact on the progression of ischemic cerebrovascular disease.

Although the patient had severe multiple vascular occlusion, dizziness as well as headache was main clinical manifestations, considering the strong compensatory mechanism of cerebral vessels mainly [12-15]: the left ICA occlusion, M1 segment of left brain artery occlusion, anterior communicating artery are open, and the right ICA system compensates the left cerebral anterior artery and middle cerebral artery through anterior communicating artery. The meningeal branch of left anterior cerebral artery supplied blood to left middle cerebral artery, and the signal of left middle cerebral artery M2 segment was low. Combined with brain CTP results, the left temporoparietal occipital lobe $\mathrm{BF}$ value was lower than the contralateral side, MTT value increased, cerebral BV compared the contralateral side was slightly elevated, considering the lack of compensation for middle cerebral artery. The patient had no symptoms such as right hemianopia, limb hemiplegia, and par- 

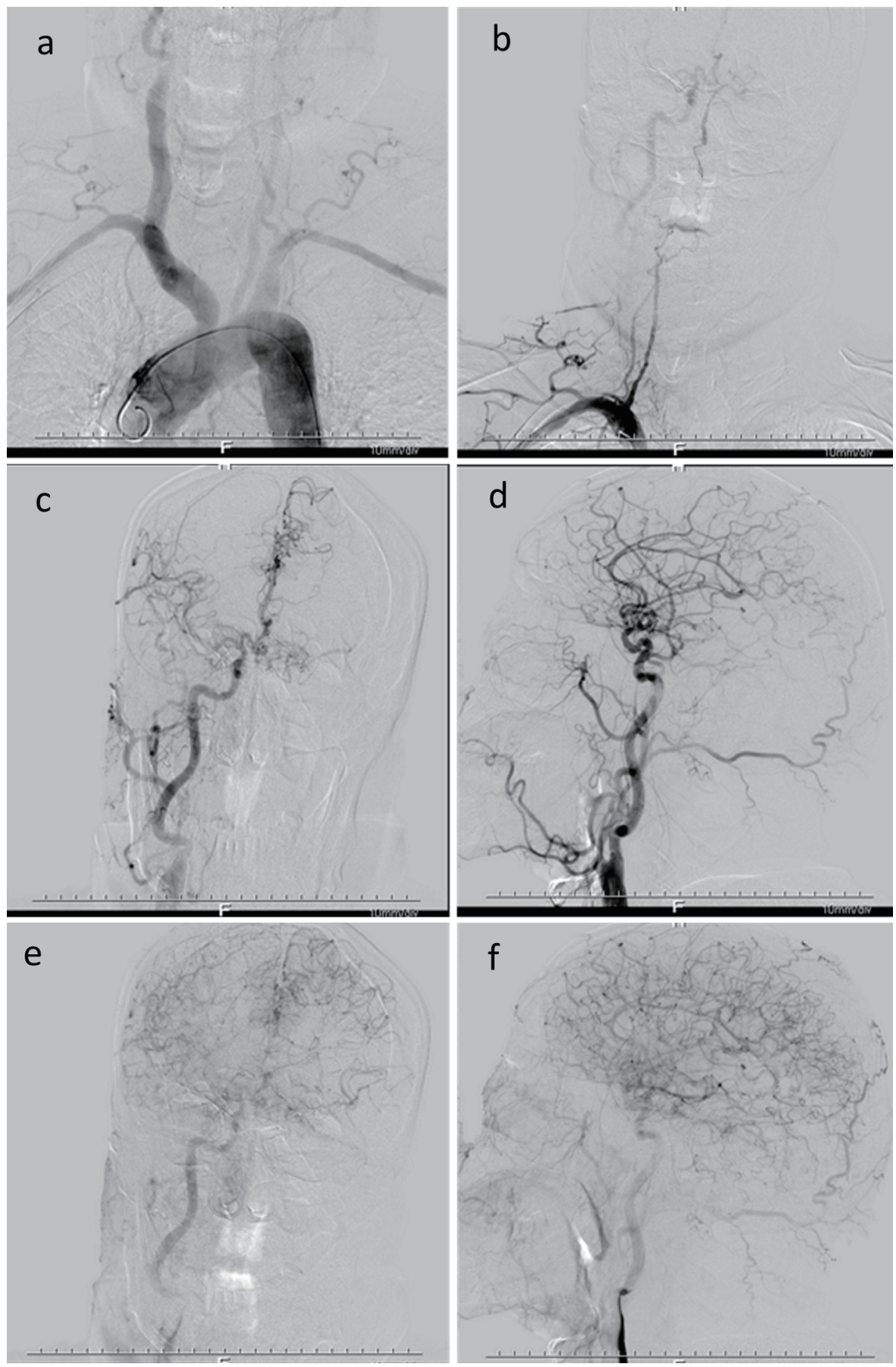

Figure 2. Multiple large vessels occlusion occurred. The right internal carotid artery system compensated blood supply to left anterior cerebral artery and middle cerebral artery through anterior communicating artery, and left anterior cerebral artery and its meningeal branch compensated blood supply to left middle cerebral artery.

tial sensory disturbance. The collateral circulation was considered because local capillary hyperplasia was observed during DSA. The V2 segment of the right vertebral artery was occluded, and its muscular branch and the branch of right cervical artery were anastomosed with the anterior spinal artery. The blood supply to the V4 segment of right vertebral artery was compensated by the anterior spinal artery, the terminal block on the left side of vertebral artery V3, no obvious compensation was found. In addition, the right posterior communicating artery was not open, basilar artery and bilateral posterior cerebral arteries were lightly developed. Therefore, the posterior circulation compensation was insufficient so that the patient's dizziness symptoms were supported.

Multiple occlusions of large vessels in the patient did not 
result in serious loss of neurological function. Just simply considering the cerebrovascular compensatory mechanism is difficult to explain why he never showed stroke-like performance in the condition of such severe inadequate compensation of the middle cerebral artery. Despite bilateral vertebral artery occlusion, there is lack the symptoms of dizziness, nystagmus, tinnitus, deafness, Horner's sign, cerebellar ataxia, coma, atresia syndrome, soft limbs of the extremities, and so on. Therefore, can we consider the participation of hypothyroidism or related drugs of hypothyroidism in the protection of nerve function? However, this hypothesis needs to be confirmed by a large number of clinical studies.

Shortcomings and limitations of this case are as follows. Although this case was associated with multiple macrovascular occlusions with hypothyroidism, a single clinical sample was insufficient to indicate that hypothyroidism was involved in the progression of vascular occlusion and individual differences caused by genetic and other factors could not be excluded. Although there is no serious neurological deficit in this patient, long-term follow-up is needed to track prognosis. Nervous system symptoms of patients with hypothyroidism can also be manifested as dizziness and headache, whose symptoms are difficult to be explained by inadequate compensation.

Atherosclerosis is affected by many factors, hypothyroidism can accelerate it, and severe vascular occlusion can occur. Taking the control of hypothyroidism actively helps to slow the progression of atherosclerosis, thereby reducing the incidence of ischemic stroke. Whether the hypothyroidism or hypothyroidism-related medication (Euthyrox) has a protective effect on the central nervous system remains to be further studied and explored.

\section{Acknowledgments}

We are grateful to the medical staff members who have treated this patient carefully.

\section{Financial Disclosure}

This is a clinical observation article without any funding.

\section{Conflict of Interest}

The authors declare that they have no conflict of interest.

\section{Informed Consent}

We obtained the patient's informed consent.

\section{Author Contributions}

YB summed up and wrote the article; QL wrote the article; SX, YW, XL and GL collected the case data.

\section{References}

1. Sacco RL, Adams R, Albers G, Alberts MJ, Benavente $\mathrm{O}$, Furie K, Goldstein LB, et al. Guidelines for prevention of stroke in patients with ischemic stroke or transient ischemic attack: a statement for healthcare professionals from the American Heart Association/American Stroke Association Council on Stroke: co-sponsored by the Council on Cardiovascular Radiology and Intervention: the American Academy of Neurology affirms the value of this guideline. Stroke. 2006;37(2):577-617.

2. Campbell BC, Mitchell PJ, Kleinig TJ, Dewey HM, Churilov L, Yassi N, Yan B, et al. Endovascular therapy for ischemic stroke with perfusion-imaging selection. N Engl J Med. 2015;372(11):1009-1018.

3. Goyal M, Demchuk AM, Menon BK, Eesa M, Rempel JL, Thornton J, Roy D, et al. Randomized assessment of rapid endovascular treatment of ischemic stroke. N Engl J Med. 2015;372(11):1019-1030.

4. Lackland DT, Carey RM, Conforto AB, Rosendorff C, Whelton PK, Gorelick PB. Implications of Recent Clinical Trials and Hypertension Guidelines on Stroke and Future Cerebrovascular Research. Stroke. 2018;49(3):772779 .

5. Warsch JR, Wright CB. Stroke: hyperlipidemia and cerebral small-vessel disease. Nat Rev Neurol. 2010;6(6):307308.

6. Shah RS, Cole JW. Smoking and stroke: the more you smoke the more you stroke. Expert Rev Cardiovasc Ther. 2010;8(7):917-932.

7. Hak AE, Pols HA, Visser TJ, Drexhage HA, Hofman A, Witteman JC. Subclinical hypothyroidism is an independent risk factor for atherosclerosis and myocardial infarction in elderly women: the Rotterdam Study. Ann Intern Med. 2000;132(4):270-278.

8. Kim H, Kim TH, Kim HI, Park SY, Kim YN, Kim S, Kim $\mathrm{MJ}$, et al. Subclinical thyroid dysfunction and risk of carotid atherosclerosis. PLoS One. 2017;12(7):e0182090.

9. Kim JT, Park MS, Choi KH, Nam TS, Choi SM, Cho KH. Clinical implications of collateral middle cerebral artery flow in acute ischaemic stroke with internal carotid artery occlusion. Eur J Neurol. 2011;18(12):1384-1390.

10. Lee SB, Huh PW, Kim DS, Yoo DS, Lee TG, Cho KS. Early superficial temporal artery to middle cerebral artery bypass in acute ischemic stroke. Clin Neurol Neurosurg. 2013;115(8):1238-1244.

11. Anderson WW, Jaros RM. BASILAR ARTERY DISEASE-Clinical Manifestations. Calif Med. 1960;92(6):400-402.

12. Nakayama H, Jorgensen HS, Raaschou HO, Olsen TS. Compensation in recovery of upper extremity function after stroke: the Copenhagen Stroke Study. Arch Phys Med Rehabil. 1994;75(8):852-857.

13. Huisa BN, Neil WP, Schrader R, Maya M, Pereira B, Bruce NT, Lyden PD. Clinical use of computed tomographic perfusion for the diagnosis and prediction of lesion growth in acute ischemic stroke. J Stroke Cerebrovasc Dis. 2014;23(1):114-122. 
14. Marcolini Evie. Brain trust: CTP improves outcomes in large vessel occlusion acute stroke. Emergency Medicine News. 2017;39(2):10.
15. Shen J, Li X, Li Y, Wu B. Comparative accuracy of CT perfusion in diagnosing acute ischemic stroke: A systematic review of 27 trials. PLoS One. 2017;12(5):e0176622. 